\title{
Isabelle Agier
}

\section{Credit officers' ability and gender discrimination: Evidence from microcredit in Rio de Janeiro's slums}

\section{TESE DE DOUTORADO}

\author{
DEPARTAMENTO DE ECONOMIA
}

Postgraduate program in Economics 


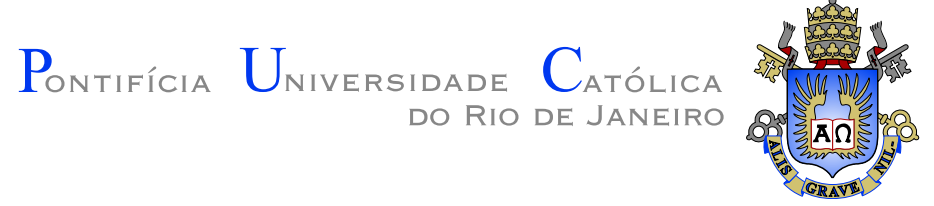

Isabelle Agier

\section{Credit officers' ability and gender discrimination: Evidence from microcredit in Rio de Janeiro's slums}

Tese de Doutorado

Thesis presented to the Postgraduate Program in Economics of the Departamento de Economia, PUC-Rio as partial fulfillment of the requirements for the degree of Doutor em Economia

Advisor: Prof. Juliano Junqueira Assunção

Rio de Janeiro

July 2010 


\title{
Isabelle Agier
}

\section{Credit officers' ability and gender discrimination: Evidence from microcredit in Rio de Janeiro's slums}

Thesis presented to the Postgraduate Program in Economics of the Departamento de Economia, PUC-Rio as partial fulfillment of the requirements for the degree of Doutor em Economia. Approved by the following commission:

\author{
Prof. Juliano Junqueira Assunção \\ Advisor \\ Departamento de Economia - PUC-Rio \\ Prof. Ana Carla Abrao Costa \\ Itau Unibanco \\ Prof. Marcelo Cortes Neri \\ EPGE-FGV
}

Prof. Rodrigo Reis Soares

Departamento de Economia — PUC-Rio

Prof. João Manoel Pinho de Mello

Departamento de Economia - PUC-Rio

Prof. Mônica Herz

Coordinator of the Centro de Ciências Sociais - PUC-Rio

Rio de Janeiro - July 08, 2010 
All rights reserved.

\section{Isabelle Agier}

Isabelle Agier graduated in Econometrics from Université de Lille 1 in 2001. She obtained a Master degree in Statistics from Ecole Nationale de la Statistique et de l'Administration Economique (Paris, France) and in Economics from Paris School of Economics in 2004.

Bibliographic data

Agier, Isabelle

Credit officers' ability and gender discrimination: Evidence from microcredit in Rio de Janeiro's slums / Isabelle Agier ; advisor: Juliano Junqueira Assunção. - 2010.

136 f. : il. ; $30 \mathrm{~cm}$

Tese (Doutorado em Economia)-Pontifícia Universidade Católica do Rio de Janeiro, Rio de Janeiro, 2010.

Inclui bibliografia

1. Economia - Teses. 2. Microfinanças. 3. Analista de crédito. 4. Gênero. 5. Discriminação. I. Assunção, Juliano. II. Pontifícia Universidade Católica do Rio de Janeiro. Departamento de Economia. III. Título. 


\section{Acknowledgments}

Para essa tese quero agradecer no Brasil,

Ao Juliano Assunção, meu orientador, para seu apoio para entrar no doutorado, e para seu entusiasmo e seus conselhos ao longo dessa tese.

Ao Teófilo Cavalcanti, superintendente do Vivacred, que me deu toda a sua confiança, me dando acesso a esta fabulosa base de dados, resultado de muito trabalho da equipe toda.

À equipe do Vivacred que me acolheu, me integrou, e respondeu a todas as minhas perguntas com paciência e bom humor. Aos clientes que aceitaram a minha presença sempre com muita gentileza. Espero que essa tese possa contribuir ao constante esforço de reflex ao da equipe que sempre preocupa melhorar seus métodos de trabalho ao serviço dos clientes.

A todas as pessoas que, durante as minhas apresentações, fizeram comentários preciosos para melhorar o meu trabalho: os professores da PUC, os pesquisadores do Banco Central, os alunos da pós e, em particular, do microtalk. Gostaria de agradecer ao Leonardo Rezende para seu apoio técnico.

Aos membros da minha banca, que aceitaram, com muita gentileza, contribuir a enriquecer a versão final dessa tese.

À CAPES e a FAPERJ pelos auxílios concedidos para essa tese.

En Europe, je voudrais adresser mes plus chaleureux remerciements,

A Ariane Szafarz, co-directrice du CERMi (Belgique), pour m'avoir guidé pendant la seconde partie de ma thèse, pour ses conseils, sa grande disponibilité et pour tout son soutien. Merci aussi pour la transition de l'après thèse.

Aux membres du CERMi et du CEB qui m'ont accueillis avec beaucoup de gentillesse et pour leurs commentaires lors de mes présentations, en particulier à Marc Labie et Mareck Hudon. Je remercie également tous ceux qui ont pris le temps d'envoyer leurs remarques.

In both side of the Atlantic, for an helpful editing, Chris, Jamie, Nacim, Marie, Hamilton, thank you so much! All remaining mistakes are mine.

Enfin, un immense merci à mon supporter du quotidien pour son appui technique, son soutien moral et ses bons petits plats ... 


\section{Abstract}

Agier, Isabelle; Assunção, Juliano (Advisor). Credit officers' ability and gender discrimination: Evidence from microcredit in Rio de Janeiro's slums. Rio de Janeiro, 2010. 136p. Tese de Doutorado Departamento de Economia, Pontifícia Universidade Católica do Rio de Janeiro.

This thesis studies the influence of credit officer in the mechanisms of microcredit based on a database from Vivacred, an NGO operating in Rio de Janeiro's slums. A brief history of Vivacred and microcredit in Brazil is presented in chapter two. Chapter three estimates a structural model and show credit officer's ability is more heterogeneous in selection that audit phase. Furthermore, officer's ability is correlated with the internal experience, but not with the previous one, showing the importance of human resource management. As credit officers have a relative autonomy, the remaining chapters analyze whether this translates into discriminatory behavior toward women. The fourth chapter documents that women enjoy equal access to credit, but obtain lower volumes than men. The officer (respectively the credit committee) is responsible for $27.3 \%$ (resp. $7.4 \%$ ) of the difference in loan size between genders, while $65.3 \%$ is attributable to the women themselves as they request significantly lower amounts. The fifth chapter compares genders in terms of delay, default and loss. For the same loan size, women repay better than men, in the three dimensions, implying that the difference found in the previous chapter is not economically justified. Moreover, the gender-gap is not reduced along the relationship, as it would be expected if its origin was asymmetric information. A theoretical model à la Becker, the sixth chapter, shows that discrimination can lead to three possible situations: the rejection of female applicants, loan downsizing for female or no impact. Empirical results confirm the second case. We tend to attribute these results to cultural habits. Women lack credibility, especially among the most ambitious ones. We do not believe that this phenomenon is necessarily conscious or volunteer. This work simply aims at contibuting to a reflexion on fair attendance.

\section{Keywords}

Microfinance. Credit officer. Gender. Discrimination. 


\section{Resumo}

Agier, Isabelle; Assunção, Juliano (Orientador). Habilidade dos analistas de crédito e discriminação de gênero: Evidencias do microcrédito em favelas do Rio de Janeiro. Rio de Janeiro, 2010. 136p. Tese de Doutorado - Departamento de Economia, Pontifícia Universidade Católica do Rio de Janeiro.

Esta tese estuda a influência do analista de crédito nos mecanismos de concessão de microcrédito a partir de uma base de dados do Vivacred, uma ONG que atua em favelas do Rio de Janeiro. Um breve histórico do Vivacred e do microcrédito no Brasil é apresentado no segundo capítulo. 0 terceiro capítulo estima um modelo estrutural cujos resultados revelam que a heterogeneidade na habilidade dos analistas é mais importante na fase de seleção que na fase de auditoria dos projetos. Além disso, a habilidade dos analistas é correlacionada com a experiência interna, mas não com a anterior, mostrando a importância da gestão dos recursos humanos. Como os analistas têm uma relativa autonomia, os demais capítulos analisam se esta se traduz em comportamentos discriminatórios em relação às mulheres. O quarto capítulo documenta que as mulheres se beneficiam de um acesso equitativo ao crédito, mas obtêm menores volumes em relação aos homens. Ao analista pode ser imputado 27,3\% da diferença no tamanho de empréstimo entre gêneros, enquanto ao comitê de crédito, apenas 7,4\%; no entanto, 65,3\% é imputável às próprias mulheres que pedem empréstimos menores. O quinto capítulo compara gêneros em termo de atraso, inadimplência e perda. Para o mesmo montante de empréstimo, a mulher tem melhor reembolso em relação aos homens, nas três dimensões, implicando que o diferencial encontrado no capítulo anterior não é justificável economicamente. Além disso, o diferencial não se reduz ao longo do relacionamento, conforme seria esperado caso sua origem fosse a informação assimétrica. Um modelo teórico à la Becker, no sexto capítulo, mostra que discriminação pode levar a três possíveis estados: a rejeição de candidaturas femininas, a redução de empréstimos para maiores pedidos femininos ou a nenhum impacto. Resultados empíricos confirmam o segundo caso. Tendemos a atribuir os presentes resultados aos hábitos culturais. Às mulheres falta credibilidade, especialmente entre as mais ambiciosas. Não acreditamos que esse fenômeno seja voluntário ou consciente e queremos simplesmente alimentar a reflexão a respeito de um atendimento equitativo.

\section{Palavras-chave}

Microfinanças. Analista de crédito. Gênero. Discriminação. 


\section{Contents}

1 Introduction $\quad 8$

2 Microfinance in Brazil $\quad 14$

3 The role of credit officers $\quad 22$

3.1 Introduction 22

3.2 Credit officer's role in credit cycle 24

$\begin{array}{lll}3.3 & \text { Data description } & 26\end{array}$

3.4 Credit officers' ability and loan performance 31

3.5 Credit officers matters more for selection 36

3.6 Vivacred-specific experience vs previous skills 41

3.7 Simulations: Eliminating heterogeneity 43

3.8 Conclusion 49

$4 \quad$ Gender-specific treatment $\quad 50$

4.1 Introduction $\quad 50$

4.2 Data 53

4.3 Gender-specific Approval and Loan Size $\quad 58$

4.4 Who is responsible for loan downsizing? $\quad 66$

$\begin{array}{ll}4.5 \text { Conclusion } & 71\end{array}$

5 The Curse of the Trustworthier Sex $\quad 74$

5.1 Introduction 74

5.2 Data and Methodology 77

$\begin{array}{lll}5.3 & \text { Empirical Results } & 82\end{array}$

5.4 What is a woman's involvement worth? 89

5.5 Gender-gap nature: Occupationnal choice 92

5.6 Conclusion 93

6 Is There a Glass Ceiling in Loan Size? $\quad 96$

$\begin{array}{lll}6.1 & \text { Introduction } & 96\end{array}$

$\begin{array}{lll}6.2 & \text { The Model } & 98\end{array}$

6.3 Glass ceiling in Loan Size for Women 103

6.4 Gender-Affinity between Clients and Officers? 108

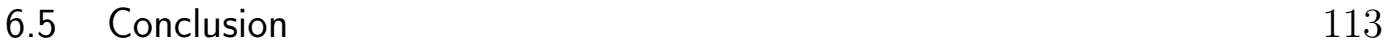

$\begin{array}{llr}7 & \text { Conclusion } & 116\end{array}$

$\begin{array}{lr}\text { Bibliography } & 127\end{array}$

$\begin{array}{lll}\text { A Appendix of Chapter } 3 & 128\end{array}$

B Appendix of Chapter $4 \quad 132$

$\begin{array}{ll}\text { C Appendix of Chapter } 5 & 134\end{array}$

$\begin{array}{lll}\text { D Appendix of Chapter } 6 & 136\end{array}$ 